\title{
CHRONIC OSTEOMYELITIS AS A COMPLICATION FROM MYCOBACTERIOSIS AFTER BREAST IMPLANT
}

Márcia Portela Melo¹, Luiza Guimaraes de Magdalena', Marcelo Wainberg Jeffman¹, Murilo de Lima Brazan¹, Jose Antonio Crespo Cavalheiro ${ }^{1}$

${ }^{1}$ Hospital de Clínicas de Porto Alegre - Porto Alegre (RS), Brazil.

Introduction: Infection is one of the most feared complications in surgeries with the inclusion of breast implants, associated with the need for new procedures and aesthetic compromise. The agents that are most associated with this conditions are the ones that colonize the skin, such as Staphylococcus aureus and Coagulase-negative staphylococci. Case Report: We described the case of a 25-year old patient, who came to our service after an augmentation mammoplasty performed about 60 days before in a hospital in the countryside. She reported bilateral seroma and extrusion of the right breast implant on the $14^{\text {th }}$ postoperative day, being submitted to antibiotic therapy and a new procedure for site review, with the placement of new implants, bilaterally. When she came to our service, on the $45^{\text {th }}$ day after the exchange of implants, she presented with pain, fever, hyperemia and drainage of the purulent secretion in the operative wounds with extrusion of the implants. In the transoperative period, after the changes were identified, on the $3^{\text {rd }}, 4^{\text {th }}$ and $5^{\text {th }}$ costal arches to the right, suggestive of osteomyelitis, associated with partial necrosis of the intercostal muscles and pectoralis minor. The bacteriological test of the periprosthetic secretion was bilaterally positive for mycobacteruim abscessus, and the anatomopathological study of the right costal arch biopsy was compatible with chronic osteomyelitis. The patient presented favorable clinical evolution after the removal of the implants and antibiotic therapy. Discussion: Mycobacteria are opportunistic organisms, which hardly cause diseases in human beings. Despite being uncommon, the infection of breast implants by mycobacteria can be associated with the contamination of surgical instruments and immunosuppression of the host. The infection can be similar to those caused by more common agents. In many cases, it presents with the later onset of symptoms. Prophylaxis and antisepsis, including special care regarding the protocols of sterilization of surgical instruments, are still the main factors associated with the impact of this type of complication in surgeries with breast implants. 